TRANSACTIONS OF THE

AMERICAN MATHEMATICAL SOCIETY

Volume 272, Number 1, July 1982

\title{
TWO CONSEQUENCES OF DETERMINACY CONSISTENT WITH CHOICE
}

\author{
BY
}

JOHN R. STEEL AND ROBERT VAN WESEP

\begin{abstract}
We begin with a ground model satisfying $\mathrm{ZF}+\mathrm{AD}+\mathrm{AC}_{\mathbf{R}}$, and from it construct a generic extension satisfying $\mathrm{ZFC}+\boldsymbol{\delta}_{2}^{1}=\omega_{2}+$ "the nonstationary ideal on $\omega_{1}$ is $\omega_{2}$-saturated".
\end{abstract}

0. Introduction. The "axiom" of determinacy (henceforth AD) implies many interesting propositions about small cardinal numbers, for example $\omega_{1}$ and $\omega_{2}$. It is natural to ask whether these propositions are true or, more realistically, can be true in the full universe $V$ of sets. Our working hypothesis is that in $V$ the axiom of choice is true and all definable games are determined, so the question becomes whether these propositions are consistent with ZFC together with definable determinacy. (We shall be vague about "definable" when we can afford to.) In this paper we shall consider two such consequences of $A D$, the first being that $\delta_{2}^{1}=\omega_{2}$, and the second that the nonstationary ideal on $\omega_{1}$ is $\omega_{2}$-saturated. We shall show that the theory consisting of $\mathrm{ZFC}+\mathrm{AD}^{L(R)}$ together with these two propositions is consistent. $\left(\mathrm{AD}^{L(\mathbf{R})}\right.$ is the assertion that all games in $L(\mathbf{R})$, a large class of definable games, are determined.)

In consonance with our working hypothesis, we would like to prove our result assuming only the consistency of $\mathrm{ZFC}+\mathrm{AD}^{L(\mathrm{R})}$, or equivalently, that of $\mathrm{ZF}+\mathrm{AD}$ + DC (cf. §1). At present we cannot do this. We instead assume the consistency of $\mathrm{ZF}+\mathrm{AD}+\mathrm{AC}_{\mathbf{R}}$, where $\mathrm{AC}_{\mathbf{R}}$ is the axiom of choice for families indexed by the reals. Consistency-wise, $\mathrm{ZF}+\mathrm{AD}+\mathrm{AC}_{\mathbf{R}}$ is one of the strongest theories known to man; we show in $\$ 1$ that it proves the consistency of $\mathrm{ZF}+\mathrm{AD}+\mathrm{DC}$. The only upper bound we know on the consistency strength of $Z F+A D+A C_{R}$ is that of $\mathrm{ZF}+\mathrm{AD}_{\mathbf{R}}+$ " $\theta$ is regular". We offer some partial justification for our use of such a strong hypothesis at the end of the paper.

Our consistency proof is by forcing. We start with a ground model $M$ satisfying $\mathrm{ZF}+\mathrm{AC}+\mathrm{AC}_{\mathbf{R}}$. By a two-step iteration we construct a generic extension $M[G]$ so that $\exists h\left(h: \omega_{2}^{M} \stackrel{\text { onto }}{\rightarrow} \mathbf{R}^{M}\right.$ ). The forcing will add no new reals and preserve $\omega_{2}$, i.e., $\mathbf{R}^{M}=\mathbf{R}^{M[G]}$ and $\omega_{2}^{M}=\omega_{2}^{M[G]}$. We then pass to the inner model $N=L\left(\mathbf{R}^{M}, h\right)$ and show it has the desired properties.

The paper is organized as follows: In $\S 1$ we give some preliminaries and background information. In particular, we set forth those consequences of $\mathrm{AD}$ whose

Received by the editors February 18, 1980.

1980 Mathematics Subject Classification. Primary 03E35; Secondary $03 E 60$.

(C)1982 American Mathematical Society 0002-9947/81/0000-1000/\$05.75 
truth in $M$ will be useful to us. Except for some scattered observations and 1.3.3, none of this material is new. In $\$ 2$ we construct $M[G]$ and prove the facts about it stated above; it follows at once that $N \vDash \mathrm{ZFC}+\mathrm{AD}^{L(\mathbf{R})}+\delta_{2}^{1}=\omega_{2}$. In $\S 3$ we show that in $N$ the nonstationary ideal on $\omega_{1}$ is $\omega_{2}$-saturated. In $\$ 4$ we discuss some of the open problems raised by this work.

\section{Preliminaries.}

1.1. Some consequences of AD. Given sets $X$ and $Y$, we regard ${ }^{X} Y=\{f \mid f: X \rightarrow Y\}$ as a topological space with the topology generated by basic open sets of the form

$$
N_{s}=\left\{f \in{ }^{x} Y \mid s \subseteq f\right\},
$$

where $s$ maps a finite subset of $X$ into $Y$. If $f$ is a bijection between $X$ and $X^{\prime}$ and $g$ a bijection between $Y$ and $Y^{\prime}$, then from the pair $(f, g)$ we can define a homeomorphism between ${ }^{X} Y$ and ${ }^{X^{\prime}} Y^{\prime}$. We are interested in the case $X$ and $Y$ are countably infinite, so that ${ }^{X} Y$ is homeomorphic to ${ }^{\omega} \omega$. We define $\mathbf{R}={ }^{\omega} \omega$, and call the elements of $\mathbf{R}$ reals. One advantage of $\mathbf{R}$ over the usual reals is that it is homeomorphic to any countable product of copies of itself.

A set of reals $A$ is comeager iff $\bigcap_{n<\omega} U_{n} \subseteq A$ for some sequence $\left\langle U_{n} \mid n<\omega\right\rangle$ of open dense sets. $A$ is meager iff $\mathbf{R}-A$ is comeager. The Baire category theorem states that no meager set is comeager.

A set of reals $A$ has the Baire property (or is "almost open") iff $\exists \mathscr{Q}$ ( $\mathscr{Q}$ is open and $A \triangle U$ is meager). A diagonal construction using a wellorder of $\mathbf{R}$ easily produces a set of reals without the Baire property. The use of a wellorder of $\mathbf{R}$ is unavoidable, and in fact

Theorem 1.1.1 (Banach, Mazur). Assume $Z F+A D+D C$. Then every set of reals has the Baire property.

We remark in passing that if every set of reals has the Baire property, then the axiom of choice for families of two-element sets fails. Specifically, there is no function picking a member from each unordered pair of Turing degrees. (A similar example was discovered long ago by Sierpinski.)

The notion of size provided by Baire category behaves much like Lebesgue measure. In particular, we have an analog of Fubini's Theorem.

TheOREM 1.1.2 (Kuratowski, Ulam). Let $S \subseteq \mathbf{R} \times \mathbf{R}$ have the Baire property. Then $S$ is comeager iff $\{x \mid\{y \mid\langle x, y\rangle \in S\}$ is comeager $\}$ is comeager.

If all sets of reals have the Baire property, then 1.1.2 yields the following useful corollary.

COROLlaRY 1.1.3 (Folk?). Assume all sets of reals have the Baire property, and let $\left\langle A_{\xi}\right| \xi\langle\kappa\rangle$ be a wellordered sequence of comeager sets. Then $\cap_{\xi<\kappa} A_{\xi}$ is comeager.

We turn now to ordinal measures of the size of $\mathbf{R}$. We must be careful in defining such measures, since AD implies every wellordered set of reals is countable.

DEFINITION 1.1.4.

$$
\boldsymbol{\theta}=\sup \{\alpha \in O R \mid \exists f(f: \mathbf{R} \stackrel{\text { onto }}{\rightarrow} \alpha)\}
$$


Clearly $\theta$ is a cardinal. Results of $\mathrm{H}$. Friedman and Y. Moschovakis show that, given $\mathrm{AD}+\mathrm{DC}, \boldsymbol{\theta}$ is quite large.

Theorem 1.1.5 (Moschovakis [6]). Assume $Z F+A D+D C$. Then $\theta$ is a limit of cardinals which are weakly inaccessible and measurable.

We remark that $\mathrm{AD}$ implies there are measurable successor cardinals, the first two being $\omega_{1}$ and $\omega_{2}$.

Clearly, $\mathrm{DC}$ implies $\operatorname{cof}(\theta)>\omega$. If $\mathrm{ZF}+\mathrm{AD}+\mathrm{DC}$ is consistent, then so is $\mathrm{ZF}+\mathrm{AD}+\mathrm{DC}+\theta$ regular (cf. 1.2.2). On the other hand, Solovay has shown in [8] that $\mathrm{ZF}+\mathrm{AD}+\mathrm{DC}+\operatorname{cof}(\theta)=\omega_{1}$ is consistent, granted the consistency of $\mathrm{ZF}+$ $\mathrm{AD}_{\mathbf{R}}+\boldsymbol{\theta}$ is regular (a theory of much greater consistency strength than $\mathrm{ZF}+\mathrm{AD}$ $+\mathrm{DC})$.

The regularity of $\theta$ is equivalent to a certain choice principle. For $B \subseteq \mathbf{R}^{3}$, we let $B_{x, y}=\{z \mid\langle x, y, z\rangle\} \in B$.

DeFinition 1.1.6. Collection is the assertion

$$
(\forall x \in \mathbf{R} \exists A \subseteq \mathbf{R}\langle x, A\rangle \in U) \Rightarrow\left(\exists B \in \mathbf{R}^{3} \forall x \in \mathbf{R} \exists y \in \mathbf{R}\left\langle x, B_{x, y}\right\rangle \in U\right) .
$$

Theorem 1.1.7 (Solovay [8]). Assume $Z F+A D+D C$. Then Collection holds iff $\theta$ is regular.

By effectivizing the definition of $\theta$, we arrive at the projective ordinals. More precisely

Definition 1.1.8. Let $n \in \omega$. Then

$$
\boldsymbol{\delta}_{n}^{1}=\sup \left\{\alpha \in O R \mid \exists f\left(f: \mathbf{R}^{\text {onto }} \rightarrow \alpha \wedge\{\langle x, y\rangle \mid f(x) \leqslant f(y)\} \text { is } \Delta_{n}^{1}\right)\right\} .
$$

During the past 15 years, descriptive set theorists have devoted much effort to determining the size of the $\boldsymbol{\delta}_{n}^{1}$ 's, assuming definable determinacy where necessary. We shall not attempt to summarize this work, but only mention that part of it is directly useful in motivating or carrying out the work of $\$ 2$. We refer the reader to [4] for more information.

The work in question led to, and then was motivated by, the following attractive conjecture.

Conjecture (D. Martin). $\forall n \geqslant 1\left(\delta_{n}^{1}=\omega_{n}\right)$.

This is meant as a conjecture about the full universe $V$, where AC holds, rather than about some inner model of $\mathrm{AD}$. The conjecture, if true, provides an explicit, definable failure of the Continuum Hypothesis $(\mathrm{CH})$.

Some of the upper bounds conjectured above for the $\delta_{n}^{1}$ 's are provable from $\mathrm{ZFC}+\mathrm{AD}^{L(\mathbf{R})}$. In fact we have

THEOREM 1.1.9. “Assume” ZFC. Then
(a) $\delta_{1}^{1}=\omega_{1}$ (Suslin);
(b) $\delta_{2}^{1} \leqslant \omega_{2}$ (Kunen, Martin);
(c) if all $\Sigma_{1}^{1}$ games are determined, then $\delta_{3}^{1} \leqslant \omega_{3}$ (Martin);
(d) if all projective games are determined, then $\forall n\left(\delta_{2 n+2}^{1} \leqslant\left(\delta_{2 n+1}^{1}\right)^{+}\right)$. Thus $\delta_{4}^{1} \leqslant \omega_{4}$ (Kunen, Martin). 
This theorem and the fact that no better upper bounds seem possible motivate Martin's conjecture. The theorem is proved in $[4, \S 9]$.

Notice that $\delta_{n}^{1}<\left(2^{\aleph_{0}}\right)^{+}$for all $n$. Thus if even $\omega_{2} \leqslant \boldsymbol{\delta}_{2}^{1}$, then $\mathrm{CH}$ fails. But all large cardinal or definable determinacy hypotheses known are consistent with $\mathrm{CH}$ (cf. 1.2.1), and so none of them imply even the lower bound $\omega_{2} \leqslant \delta_{2}^{1}$ conjectured above. In the present state of knowledge, the best one can hope for the lower bounds of Martin's conjecture is a proof that they are consistent with ZFC plus large cardinals or definable determinacy. We show this in $\$ 2$ for the bound $\omega_{2} \leqslant \delta_{2}^{1}$.

We return to consequences of full AD. Theorem 1.1.10 will be quite useful in $\S 2$, while 1.1.11 will serve as our point of departure in §3. Both are proved in [4].

Theorem 1.1.10 (Martin, Solovay). Assume $Z F+A D+D C$. Then $\delta_{2}^{1}=\omega_{2}$.

Theorem 1.1.11 (Solovay). Assume $Z F+A D+D C$. Then every subset of $\omega_{1}$ either includes or is disjoint from a closed unbounded subset of $\omega_{1}$; moreover, if $j$ is the embedding generated by the closed unbounded filter on $\omega_{1}$, then $j\left(\omega_{1}\right)=\omega_{2}$.

1.2. On $L(\mathbf{R}) . L(\mathbf{R})$ is the natural inner model of $\mathrm{ZF}+\mathrm{AD}+\mathrm{DC}$, if there is any. We can define $L(\mathbf{R})$ as the universe of sets constructible from the reals as urelements; alternatively, as the smallest model of $\mathrm{ZF}$ containing all reals and all ordinals. An easy induction on the construction of $L(\mathbf{R})$ shows that in $L(\mathbf{R})$ every set is ordinal definable from a real. Using ths coding in $L(\mathbf{R})$ of sets by ordinals and reals, together with $\mathrm{DC}_{\mathbf{R}}$ in $V$, we have that both $\mathrm{DC}$ and Collection are true in $L(\mathbf{R})$.

$\mathrm{AD}^{L(\mathbf{R})}$ is the assertion that all games in $L(\mathbf{R})$ are determined. This is a strong form of definable determinacy hopefully consistent with ZFC. By the preceding remarks, if $M$ is a model of $\mathrm{ZF}+\mathrm{AD}^{L(\mathbf{R})}+\mathrm{DC}_{\mathbf{R}}$, then $L(\mathbf{R})^{M}$ is a model of $\mathrm{ZF}+\mathrm{AD}+\mathrm{DC}+\boldsymbol{\theta}$ is regular. For the converse relative consistency result we use

THEOREM 1.2.1 (FolK). Let $M$ be a countable transitive model of $Z F+A D+D C$ $+V=L(\mathbf{R})$. Then there is a generic extension $\Re$ of $\Re$ such that

(a) $\Re=Z F C+C H$,

(b) $\mathbf{R}^{\Re}=\mathbf{R}^{\Re}$, hence $\Re \vDash A D^{L(\mathbf{R})}$, and

(c) if $\kappa \geqslant \theta^{\Re}$, then $\kappa$ is a cardinal in $\Re$ iff $\kappa$ is a cardinal in $\Re$.

Proof. In $\Re$, let $\mathbf{P}$ be $\left\{f \mid \exists \alpha<\omega_{1}(f: \alpha \rightarrow \mathbf{R})\right\}$, ordered by inclusion. Let $\Re=\Re[\mathcal{G}$, where $\mathcal{G}$ is $\mathbf{P}$-generic over $\Re$. $\mathbf{P}$ is $\omega$-closed by DC in $\Re$, so (b) holds. Since $\mathfrak{T} \vDash V=L(\mathbf{R})$ and $\mathcal{G}$ induces a wellorder of $\mathbf{R}^{\mathfrak{R}}=\mathbf{R}^{\Re}$, (a) holds as well.

We prove (c) by a possible values argument. Work in $\Re$. Let $\kappa$ be a cardinal $\geqslant \theta$, and let $f \in \mathbf{P}, \beta<\kappa$, and $\tau$ be a term so that

$$
f \Vdash \tau \text { is a map of } \check{\beta} \text { onto } \check{\kappa} \text {. }
$$

For $\delta<\beta$ let

$$
S_{\delta}=\{\gamma \mid \exists g \supseteq f(g \Vdash \tau(\check{\delta})=\check{\gamma})\} .
$$

Then $S_{\delta}$ has order type $<\theta$, since it is the surjective image of $\mathbf{P}$, which in turn is the surjective image of $\mathbf{R}$. But $\kappa=\cup_{\delta<\beta} S_{\delta}$ since $f \Vdash \tau$ is onto. In the case $\kappa>\theta$ this is an immediate contradiction, while if $\kappa=\theta$ we need only observe that $\kappa$ is regular for our contradiction. 
COROLLARY 1.2.2. The following theories are equiconsistent.

(a) $Z F+A D^{L(\mathbf{R})}+D C_{\mathbf{R}}$,

(b) $Z F C+A D^{L(\mathbf{R})}+C H$,

(c) $Z F+A D+D C$,

(d) $Z F+A D+D C+\theta$ is regular.

We do not know whether $Z F+A D$ can be added to this list.

We originally considered the work of $\S 2$ as a proof that $\mathrm{ZFC}+\mathrm{AD}^{L(\mathbf{R})}+-\mathrm{CH}$ is consistent. As such, however, it suffers two defects: first, one must assume more than the consistency of $\mathrm{ZFC}+\mathrm{AD}^{L(R)}$, and second, only the value $2^{\omega}=\omega_{2}$ is obtained. H. Friedman and H. Woodin then found a proof free of these defects, so that e.g. $\mathrm{ZFC}+\mathrm{AD}^{L(\mathbf{R})}+2^{\omega}=\omega_{17}$ may be added to the list in 1.2.2 (cf. [10]). The Friedman-Woodin method, however, gives no information as to the possible sizes of the projective ordinals.

Our second corollary of 1.2.1 illustrates in a small way the fact that finding ways of generically extending models of $\mathrm{AD}$ to models of $\mathrm{AC}$ without too much disturbance will yield theorems about the ground model (cf. also 2.1.12).

Corollary 1.2.3. Assume $Z F+A D+D C+V=L(\mathbf{R})$. Then $\forall \kappa \geqslant \theta\left(\kappa^{+}\right.$is regular).

Proof. Let $\mathfrak{N}$ be a countable transitive model of a sufficiently large fragment of $\mathrm{ZF}+\mathrm{AD}+\mathrm{DC}+V=L(\mathbf{R})$, and let $\Re$ be its extension as in 1.2.1. If $\Re \vDash \kappa \geqslant \theta \wedge$ $\boldsymbol{\kappa}^{+}$is singular, then by 1.2.1(c), $\mathfrak{N} \vDash \boldsymbol{\kappa}^{+}$is singular. But AC holds in $\mathscr{N}$.

AD implies there are singular successor cardinals below $\theta$, the least such being $\omega_{3}$.

Although our ground model will be much larger than $L(\mathbf{R})$, the next theorem implies that, below $\theta^{L(R)}$, its cardinals and cofinalities will be the same as those of $L(\mathbf{R})$.

Theorem 1.2.4 (MoschovaKis). Assume $Z F+A D+D C$. Let $\kappa<\theta^{L(\mathbf{R})}$ and $A \subseteq \kappa$. Then $A \in L(\mathbf{R})$.

Proof. Cf. [6].

We consider another choice principal hopefully consistent with $A D$, but this time false in $L(\mathbf{R})$.

DEFINITION 1.2.5. Unif $(\mathbf{R} \times \mathbf{R})$ is the assertion that every relation on reals can be uniformized. That is, Unif(R $\times \mathbf{R})$ iff $\forall\left\{A_{x} \mid x \in \mathbf{R}\right\} \exists f: \mathbf{R} \rightarrow \mathbf{R} \forall x\left(\varnothing \neq A_{x} \subseteq \mathbf{R} \Rightarrow\right.$ $\left.f(x) \in A_{x}\right)$.

TheOREM 1.2.6. Assume $Z F C+A D^{L(\mathbf{R})}$. Then $L(\mathbf{R}) \vDash-U n i f(\mathbf{R} \times \mathbf{R})$.

Proof. Work in $L(\mathbf{R})$. Let $A_{x}=\{y \mid y$ is not ordinal definable from $x\}$, for $x \in \mathbf{R}$. Then $A_{x} \neq \varnothing$ since $\mathbf{R}-A_{x}$ is wellorderable. Suppose $f$ were a choice function for $\left\{A_{x} \mid x \in \mathbf{R}\right\}$. Since $f \in L(\mathbf{R}), f$ is ordinal definable from some $x_{0} \in \mathbf{R}$. But then $f\left(x_{0}\right) \notin A_{x_{0}}$, a contradiction.

1.3. Stronger than AD. We shall assume our ground model satisfies $\mathrm{ZF}+\mathrm{AD}+$ $\mathrm{DC}+\mathrm{AC}_{\mathbf{R}}$.

Definition 1.3.1. $\mathrm{AC}_{\mathbf{R}}$ is the assertion: $\forall\left\{A_{x} \mid x \in \mathbf{R}\right\} \exists f: \mathbf{R} \rightarrow \mathbf{R} \forall x\left(A_{x} \neq \varnothing \Rightarrow\right.$ $\left.f(x) \in A_{x}\right)$. 
So $\operatorname{Unif}(\mathbf{R} \times \mathbf{R})$ is $\mathrm{AC}_{\mathbf{R}}$ restricted to families of sets of reals. Besides Unif $(\mathbf{R} \times \mathbf{R}), \mathrm{AC}_{\mathbf{R}}$ also implies $\theta$ is regular. On the other hand, if $\mathrm{ZF}+\mathrm{AD}+$ $\mathrm{Unif}(\mathbf{R} \times \mathbf{R})+\theta$ is regular is consistent, then so is $\mathrm{ZF}+\mathrm{AD}+\mathrm{DC}+\mathrm{AC}_{\mathbf{R}}$. We prove this by considering the inner model $L(\mathbf{R} \cup P(\mathbf{R}))$, the universe of sets constructible from the reals and sets of reals as urelements.

Here and later the notion of Wadge reducibility will be useful. For $A, B \subseteq \mathbf{R}$, let $A \leqslant_{w} B$ iff $\exists f: \mathbf{R} \rightarrow \mathbf{R}$ ( $f$ is continuous $\wedge A=f^{-1}(B)$ ). AD directly implies that $\forall A, B \subseteq \mathbf{R}\left(A \leqslant_{w} B\right.$ or $\left.B \leqslant_{w} \mathbf{R}-A\right)$. D. Martin showed, in $\mathrm{ZF}+\mathrm{AD}+\mathrm{DC}_{\mathbf{R}}$, that $\leqslant_{w}$ is well founded, so that $\leqslant_{w}$ becomes a prewellorder after one identifies $A$ with $\mathbf{R}-A$. Let $O T_{w}(A)$ be the ordinal of $A$ in this prewellorder. Solovay showed in $\mathrm{ZF}+\mathrm{AD}+\mathrm{DC}_{\mathbf{R}}$ that $\theta=\sup \left\{O T_{w}(A) \mid A \subseteq \mathbf{R}\right\}$ (cf. [8]). This implies that for $\kappa<\theta,\left\{A \mid O T_{w}(A)<\kappa\right\}$ is the surjective image of $\mathbf{R}$. (These facts, by the way, yield at once the nontrivial direction of 1.1.7.) For more on $\leqslant_{w}$, see [9].

TheOrem 1.3.2. Assume $Z F+A D+U n i f(\mathbf{R} \times \mathbf{R})+\theta$ is regular. Then

$$
L(\mathbf{R} \cup P(\mathbf{R})) \vDash Z F+A D+D C+A C_{\mathbf{R}} \text {. }
$$

Proof. Every set in $L(\mathbf{R} \cup P(\mathbf{R}))$ is ordinal definable from a set of reals, so in verifying $\mathrm{AC}_{\mathbf{R}}$ we may assume $\forall x\left(A_{x} \subseteq P(\mathbf{R})\right)$. But then, since $\theta$ is regular, $\exists \kappa<\theta$ so that

$$
\forall x \in \mathbf{R}\left(A_{x} \neq \varnothing \Rightarrow \exists B \in A_{x}\left(O T_{w}(B)<\kappa\right)\right) .
$$

Since $\left\{B \mid O T_{w}(B)<\kappa\right\}$ is the surjective image of $\mathbf{R}$, Unif $(\mathbf{R} \times \mathbf{R})$ gives the desired choice function.

To verify $\mathrm{DC}$, it is enough to verify $\mathrm{DC}_{P(\mathbf{R})}$. Suppose $\forall A \subseteq \mathbf{R} \exists B \subseteq \mathbf{R}(\langle A, B\rangle \in$ $S)$. Since $\theta$ is regular and $\omega<\theta, \exists \kappa<\theta$ so that

$$
O T_{w}(A)<\kappa \Rightarrow \exists B\left(O T_{w}(B)<\kappa \wedge\langle A, B\rangle \in S\right) .
$$

But $\left\{B \mid O T_{w}(B)<\kappa\right\}$ is the surjective image of $\mathbf{R}$, so $\mathrm{DC}_{\mathbf{R}}$ gives a DC sequence for $S$. (Note Unif $(\mathbf{R} \times \mathbf{R}) \Rightarrow \mathrm{DC}_{\mathbf{R}}$ ).

Thus $\mathrm{ZF}+\mathrm{AD}+\mathrm{AC}_{\mathbf{R}}$ is equiconsistent with $\mathrm{ZF}+\mathrm{AD}+\operatorname{Unif}(\mathbf{R} \times \mathbf{R})+\boldsymbol{\theta}$ is regular, and $\mathrm{DC}$ can be added to either theory without increasing its consistency strength. We conclude by showing $\mathrm{ZF}+\mathrm{AD}+\mathrm{Unif}(\mathbf{R} \times \mathbf{R})$ proves the consistency of $\mathrm{ZF}+\mathrm{AD}+\mathrm{DC}$. This follows at once from 1.2.6 and the next theorem.

Theorem 1.3.3. Assume $Z F+A D+D C_{\mathbf{R}}+\exists A \subseteq \mathbf{R}(A \notin L(\mathbf{R}))$. Then there is a nontrivial elementary $j: L(\mathbf{R}) \rightarrow L(\mathbf{R})$, and so $Z F+A D+D C$ is consistent.

Proof. If $A \subseteq \mathbf{R}$ and $A \notin L(\mathbf{R})$, then $\theta^{L(\mathbf{R})}<O T_{w}(A)$. Thus $\theta^{L(\mathbf{R})}<\theta$. By 1.1.5, let $\kappa$ be measurable with $\theta^{L(\mathbf{R})}<\kappa<\theta$. Let $U$ be a normal ultrafilter on $\kappa$ and consider the ultrapower

$$
\left({ }^{\kappa} L(\mathbf{R}) \cap L(\mathbf{R})\right) / U
$$

By $D C_{\mathbf{R}}$ the ultrapower is well founded. It is enough to show that kos' theorem holds for this ultrapower. This is done by the usual induction, except in the existential quantifier case, whose usual proof would require $\mathrm{AC}$ in $L(\mathbf{R})$. For this 
case, suppose that for $U$ a.e. $\alpha<\kappa$

$$
L(\mathbf{R}) \vDash \exists v \varphi\left(v, f_{1}(\alpha) \cdots f_{n}(\alpha)\right),
$$

where $f_{i} \in L(\mathbf{R}) \cap{ }^{\kappa} L(\mathbf{R})$ for $i \leqslant n$. We want an $f \in L(\mathbf{R}) \cap{ }^{\kappa} L(\mathbf{R})$ so that for $U$ a.e. $\alpha<\boldsymbol{\kappa}$

$$
L(\mathbf{R}) \vDash \varphi\left(f(\alpha), f_{1}(\alpha), \ldots, f_{n}(\alpha)\right) .
$$

Define in $L(\mathbf{R})$, for $\alpha<\kappa, A_{\alpha}=\{x \in \mathbf{R} \mid \exists v$ ( $v$ is ordinal definable from $x \wedge$ $\left.\left.\varphi\left(v, f_{1}(\alpha), \ldots, f_{n}(\alpha)\right)\right)\right\}$. We claim that there is a fixed $A \subseteq \mathbf{R}$ so that $A_{\alpha}=A$ for $U$ a.e. $\alpha$. If not, let $X=\left\{\alpha \mid \forall \beta<\alpha\left(A_{\beta} \neq A_{\alpha}\right)\right\}$; then $X \in U \cap L(\mathbf{R})$ and $\forall \alpha, \beta \in X$ $\left(\alpha \neq \beta \Rightarrow A_{\alpha} \neq A_{\beta}\right)$. Since $\theta^{L(\mathbf{R})}<\kappa$, we have a $\delta<\theta^{L(\mathbf{R})}$ so that $O T_{w}\left(A_{\alpha}\right)<\delta$ for $U$ a.e. $\alpha$, say for all $\alpha \in Y$, where $Y \in U \cap L(\mathbf{R})$. But then $X \cap Y$ is the surjective image of $\mathbf{R}$ by a map in $L(\mathbf{R})$, a contradiction since $X \cap Y$ has order type $>\theta^{L(\mathbf{R})}$.

Let $A=A_{\alpha}$ for $U$ a.e. $\alpha$. By hypothesis $A \neq \varnothing$, so let $x \in A$. Fix in $L(\mathbf{R})$-definable wellorder $W$ of the class of $V \in L(\mathbf{R})$ so that $v$ is ordinal definable in $L(\mathbf{R})$ from $x$. Then we can define in $L(\mathbf{R})$

$$
\begin{aligned}
f(\alpha) & =W-\text { least } v \text { so that } \varphi\left(v, f_{1}(\alpha), \ldots, f_{n}(\alpha)\right), \\
& =0 \text { if no such } v \text { exists, }
\end{aligned}
$$

and $f$ is as desired.

We do not know how the consistency strength of $\mathrm{ZF}+\mathrm{AD}+\mathrm{AC}_{\mathbf{R}}$ compares with that of the theories including $\mathrm{AD}_{\mathbf{R}}$ investigated in [8]. Of course $\mathrm{ZF}+\mathrm{AD}+$ $\mathrm{AC}_{\mathbf{R}}$ is consistent relative to $\mathrm{ZF}+\mathrm{AD}_{\mathbf{R}}+\boldsymbol{\theta}$ is regular by 1.3.2. It seems plausible that $\mathrm{ZF}+\mathrm{AD}_{\mathbf{R}}$ is much stronger than $\mathrm{ZF}+\mathrm{AD}+\mathrm{AC}_{\mathbf{R}}$.

Kunen's argument adapts to show that the conclusion of 1.3.3 implies that $\mathbf{R}^{\sharp}$ (the analog of $0 \#$ for $L(\mathbf{R})$; cf. [8]) exists. Recent work of Martin and Steel shows that the determinacy of games of $\mathbf{R}$ whose payoff set is $\Pi_{1}^{1}$ (when coded as a set of reals under the map $\left.{ }^{\omega} \mathbf{R} \approx \mathbf{R}\right)$ implies $\exists A \subseteq \mathbf{R}(A \notin L(\mathbf{R}))$. Thus, if we assume $\mathrm{ZF}+\mathrm{AD}$ $+\mathrm{DC}$, we have the following analog of a theorem of Martin and Harrington (cf. [3]) about $0 \#$ : $\Pi_{1}^{1}$ games on $\mathbf{R}$ are determined iff $\mathbf{R}^{\sharp}$ exists.

2. We want to extend generically a model $M$ of $\mathrm{AD}$ to a model $N$ of $\mathrm{AC}$ without adding reals or collapsing $\omega_{2}^{M}$. (This will imply $N \vDash \delta_{2}^{1}=\omega_{2}$ by 1.1.10.) To obtain AC, we must add a wellorder of $\mathbf{R}^{M}$. Moreover, since $\omega_{2}$ is the surjective image of $\mathbf{R}$ in $M$, our wellorder must have length at least $\omega_{2}$, as otherwise it will collapse $\omega_{2}$. The natural conditions for adding such a wellorder would be functions $h: \alpha \stackrel{1-1}{\rightarrow} \mathbf{R}$ for $\alpha<\omega_{2}$, ordered by inclusion. Unfortunately, when $\alpha \geqslant \omega_{1}$ there are no such $h$ in $M$. So we first fix up $M$ by adding enough $\omega_{1}$-sequences; this gives us a model $M^{*}$. We will have $\mathbf{R}^{M^{*}}=\mathbf{R}^{M}$ and $\omega_{2}^{M^{*}}=\omega_{2}^{M}$. Forcing over $M^{*}$ with the natural conditions above then gives the desired $N$.

If the second forcing is to work, we need $\omega_{1}-\mathrm{DC}$ in $M^{*}$. Now $\omega_{1}-\mathrm{DC}$ follows (for reals, anyway) from $\mathrm{AC}_{\mathbf{R}}$ plus the existence of a uniform counting of the countable ordinals, i.e. of a function $f$ so that $\forall \alpha<\omega_{1}(f(\alpha)$ maps $\omega$ onto $\alpha)$. Assuming $M \vDash \mathrm{AC}_{\mathbf{R}}$, it will be enough to add such an $f$ and preserve $\mathrm{AC}_{\mathbf{R}}$. 
Each condition for adding $f$ to $M$ consists in part of a proper initial segment of $f$, called the stem of the condition. A condition must also restrict the possible extensions of its stem, as otherwise $\omega_{2}$ will be collapsed. Now for $\alpha<\omega_{1}$, the set of possible length $\alpha$ extensions is a topological space homeomorphic to $\mathbf{R}$. We allow a condition to restrict the length $\alpha$ extensions of its stem to a comeager set in this space. The basic reason this method of restriction preserves $\omega_{2}$ is that, by 1.1.3, $M \vDash \forall h: \mathbf{R} \rightarrow \omega_{2} \exists \beta<\omega_{2} \exists C \subseteq \mathbf{R}\left(C\right.$ is comeager $\left.\wedge h^{\prime \prime} C \subseteq \beta\right)$.

We now describe the spaces we shall use. For $\alpha<\beta<\omega_{1}$, let

$$
T_{\alpha, \beta}=\underset{\alpha \leqslant \delta<\beta}{\times}{ }^{\omega}(\omega+\delta) \text {. }
$$

Let $T_{\alpha, \beta}$ have the product topology generated by the function topologies on its factors. Each $T_{\alpha, \beta}$ is homeomorphic to $\mathbf{R}$. We sometimes identify $T_{\alpha, \beta}$ with $\{f$ : $(\beta-\alpha) \times \omega \rightarrow \omega+\beta \mid \forall \delta \forall n(f(\delta, n)<\omega+\delta)\}$. If $f \in T_{\alpha, \beta}$ and $\alpha \leqslant \delta<\beta$, then we let $f_{\delta}(n)=f(\delta, n)$.

We proceed to the construction of $M^{*}$. Although we are mainly interested in a ground model $M$ of $\mathrm{ZF}+\mathrm{AD}+\mathrm{AC}_{\mathbf{R}}$, we can get underway with much weaker assumptions about $M$.

THEOREM 2.1.1. Let $M$ be a countable transitive model of $Z F+D C+\forall A \subseteq \mathbf{R}$ ( $A$ has the Baire property). Then there is a generic extension $M^{*}$ of $M$ so that

(a) ${ }^{\omega} M \cap M^{*} \subseteq M$;

(b) cardinals and cofinalities are preserved;

(c) $M^{*} \vDash \exists f \forall \alpha<\omega_{1}(f(\alpha)$ maps $\omega$ onto $\alpha)$.

If in addition $M \vDash A C_{\mathbf{R}}$ then

(d) $M^{*} \vDash A C_{\mathrm{R}}$;

(e) $M^{*} \vDash D C$.

If in addition $M \vDash A D+V=L(\mathbf{R} \cup P(\mathbf{R}))$, then

(f) $M^{*} \vDash \omega_{1}-D C$.

Proof. In $M$, we define a condition to be a pair $(f, \vec{X})$ such that $f \in T_{0, \alpha}$ for some $\alpha<\omega_{1}$, and $\vec{X}=\left\langle X_{\beta} \mid \beta<\omega_{1}\right\rangle$, where

(1) $\forall \delta<\alpha\left(f_{\delta}: \omega \stackrel{\text { onto }}{\rightarrow} \omega+\delta\right)$, and

(2) $X_{\beta} \subseteq T_{0, \beta}$ for $\beta<\omega_{1}$, and for $\beta>\alpha\left\{h \in T_{\alpha, \beta} \mid f^{\wedge} h \in X_{\beta}\right\}$ is comeager, and

(3) $\forall \beta>\alpha$

(a) $\left(\alpha \leqslant \delta<\beta \wedge f^{\wedge} g \in X_{\beta}\right) \Rightarrow g_{\delta}: \omega \stackrel{\text { onto }}{\rightarrow} \omega+\delta$,

(b) $\alpha \leqslant \delta<\beta \wedge \hat{f} g \in X_{\beta} \Rightarrow \hat{f} g \mid \delta \in X_{\delta}$,

(c) $\alpha \leqslant \delta<\beta \wedge f^{\wedge} g \in X_{\delta} \Rightarrow\left\{h \in T_{\delta, \beta} \mid \hat{f} \hat{g} h \in X_{\beta}\right\}$ is comeager.

We visualize conditions as trees. We call $f$ the stem of $(f, \vec{X})$, and $\vec{X}$ the restriction of $(f, \vec{X})$.

If $(f, \vec{X})$ and $(g, \vec{Y})$ are conditions, then $(g, \vec{Y}) \leqslant(f, \vec{X})$ iff $(f \subseteq g \wedge g \in$ $\left.X_{\operatorname{dom}(g)} \wedge \forall \beta \geqslant \operatorname{dom}(g)\left(Y_{\beta} \subseteq X_{\beta}\right)\right)$. Let $P$ be the set of conditions and $\mathbf{P}=(P, \leqslant)$.

Let $P^{\prime}=\{(f, \vec{X}) \mid(f, \vec{X})$ satisfies (1) and (2) above $\}$, and let $\mathbf{P}^{\prime}$ be $P^{\prime}$ with the obvious order extending $\leqslant$ on $P$. Then $\mathbf{P}$ is dense in $\mathbf{P}^{\prime}$; in fact if $(f, \vec{X}) \in P^{\prime}$ then $\exists \vec{Y}\left(\forall \beta\left(Y_{\beta} \subseteq X_{\beta}\right) \wedge(f, \vec{Y}) \in P\right)$. We omit the easy proof. The only nontrivial part, 
arranging that $(f, \vec{Y})$ satisfy $3(\mathrm{c})$, uses 1.1 .2 and 1.1.3. So we could have forced with $\mathbf{P}^{\prime}$; using $\mathbf{P}$ just simplifies some details. Since $\mathbf{P}$ is dense in $\mathbf{P}^{\prime}$, one need only worry in the sequel whether an alleged condition is in $\mathbf{P}^{\prime}$; if so, one can always refine its restriction to get a true condition.

Our next lemma will not be used in the proof of 2.1.1. (It will be used in the proofs of 3.1.1 and 3.1.2.) We mention it here because it looks as if it might be useful in an attempt to do without $\mathrm{AC}_{\mathbf{R}}$ in $M$ in proving our main results.

LEMMA 2.1.2. There is a function $F \in M$ such that

(i) $\operatorname{dom} F=\{(f, \vec{X}, \varphi) \mid(f, \vec{X}) \in P \wedge \exists \vec{Y}((f, \vec{Y}) \leqslant(f, \vec{X}) \wedge(f, \vec{Y}) \Vdash \varphi)\}$, and

(ii) $(f, F(f, \vec{X}, \varphi)) \leqslant(f, \vec{X})$ and $(f, F(f, \vec{X}, \varphi)) \Vdash \varphi, f o r(f, \vec{X}, \varphi) \in \operatorname{dom} F$.

Proof. Let $F(f, \vec{X}, \varphi)=\vec{Z}$, where for $\beta<\omega_{1}$,

$$
Z_{\beta}=\cup\left\{Y_{\beta} \mid(f, \vec{Y}) \leqslant(f, \vec{X}) \text { and }(f, \vec{Y}) \Vdash \varphi\right\} \text {. }
$$

If $F(f, \vec{X}, \varphi)=\vec{Z}$, then clearly $(f, \vec{Z})$ is a condition extending $(f, \vec{X})$. Suppose for a contradiction that $(f, \vec{Z}) \nVdash \varphi$. Let $(g, \vec{W}) \leqslant(f, \vec{Z})$ and $(g, \vec{W}) \Vdash-\varphi$. Then $g \in$ $Z_{\operatorname{dom}(g)}$ so $g \in Y_{\operatorname{dom}(g)}$ for some $\vec{Y}$ so that $(f, \vec{Y}) \nVdash \varphi$. Let $V_{\beta}=W_{\beta} \cap Y_{\beta}$ for $\beta<\omega_{1}$. Then $(g, \vec{V})$ is a condition extending both $(g, \vec{W})$ and $(f, \vec{Y})$, a contradiction.

Let $G$ be P-generic over $M$, and $M^{*}=M[G]$. We prove 2.1.1 in a sequence of lemmas.

It may appear at first glance that $\mathbf{P}$ is $\omega$-closed, but this is not true. One could have a decreasing sequence $\left(f_{n}, \vec{X}^{n}\right)$ in $\mathbf{P}$ so that $\cup_{n<\omega} f_{n} \notin X_{\beta}^{\circ}$, where $\beta=$ $\operatorname{dom}\left(\cup_{n} f_{n}\right)$. Our proof 2.1.1(a) relies on the fact that $X_{\beta}^{\circ}$ is fat enough that this is unusual.

Definition 2.1.3. If $t$ is a finite function with domain a subset of $\omega_{1} \times \omega$ and $\forall\langle\alpha, n\rangle \in \operatorname{dom}(t)(t(\alpha, n)<\omega+\alpha)$, then for any $\beta<\delta$ such that $\operatorname{dom}(t) \subseteq[\beta, \delta)$ $\times \omega$

$$
[t]_{\beta, \delta}=\left\{f \in T_{\beta, \delta} \mid t \subseteq f\right\} .
$$

That is, $[t]_{\beta, \delta}$ is an interval of Baire or basic clopen set in $T_{\beta, \delta}$. We drop the subscripts when they are understood.

LEMMA 2.1.4. Let $U$ be an open dense subset of $P, p=\langle f, \vec{X}\rangle$ a condition with $f \in T_{0, \alpha}$ and for $\beta>\alpha$ let $\left.\left.S_{\beta}=\left\{g \in T_{\alpha, \beta} \mid \exists \vec{Y}(<\hat{f} g, \vec{Y}) \leqslant p \wedge<\hat{f} g, \vec{Y}\right) \in U\right)\right\}$. Then $\exists \beta \forall \delta \geqslant \beta$ ( $S_{\delta}$ is comeager $)$.

Proof. Recall that a set is comeager if it is nonmeager on every interval, since all sets are almost open. For each interval $[t]$ so that $\operatorname{dom}(t) \cap(\alpha \times \omega)=\varnothing$ there is a $\beta_{t}>\sup \{\delta \mid \exists n(\langle\delta, n\rangle \in \operatorname{dom}(t))\}$ so that $S_{\beta_{t}}$ is nonmeager on [t]. (If not, let for $\operatorname{dom}(t) \subseteq \beta \times \omega$

$$
Z_{\beta}=X_{\beta}-\left(S_{\beta} \cap[t]\right)
$$

and let $Z_{\beta}=X_{\beta}$ otherwise. Now let $\langle\hat{f} g, \vec{Y}\rangle \leqslant\langle f, \vec{Z}\rangle$ and $t \subseteq g$. Then $\langle\hat{f} \hat{g}, \vec{Y}\rangle$ has no extension in $U$.)

Notice that if $\beta \geqslant \beta_{t}$, then $S_{\beta}$ is nonmeager on [t] (by 1.1.2). Now let $\beta<\omega_{1}$ be a limit ordinal so that for each interval [ $t]$ of $T_{\alpha, \beta}, \beta_{t}<\beta$. Then $S_{\beta}$ is comeager in $T_{\alpha, \beta}$, and so $S_{\delta}$ is comeager on $T_{\alpha, \delta}$ for all $\delta \geqslant \beta$ (by 1.1.2). 
LEMMA 2.1.5. $\mathbf{P}$ is $(\omega, \infty)$ distributive.

Proof. Let $\left\langle U_{n}\right| n\langle\omega\rangle$ be a sequence of open dense subsets of $\mathbf{P}$, and $p \in \mathbf{P}$. Let $S_{\beta}^{n}$ be the $S_{\beta}$ of 2.1.4 for $U_{n}$. By 2.1.4 let $\beta<\omega_{1}$ be so that $\forall n\left(S_{\beta}^{n}\right.$ is comeager in $T_{\alpha, \beta}$ ). Let $g \in \bigcap_{n} S_{\beta}^{n}$. Using 2.1 .2 (or DC in $M$ ) it is easy to find $\vec{Y}$ so that $\left\langle f^{\prime} g, \vec{Y}\right\rangle \leqslant p$ and $\left\langle f^{\hat{\prime}} g, \vec{Y}\right\rangle \in \bigcap_{n<\omega} U_{n}$.

Of course, 2.1.1(a) now follows.

LEMMA 2.1.6. $\forall \kappa\left(\left(M \vDash \kappa\right.\right.$ is a cardinal iff $M^{*} \vDash \kappa$ is a cardinal $) \wedge \operatorname{cof}^{M}(\kappa)=$ $\left.\operatorname{cof}^{M^{*}}(\kappa)\right)$.

Proof. We use a possible values argument. Suppose

$$
(f, \vec{X}) \Vdash \tau \text { maps } \check{\gamma} \text { into } \check{\kappa},
$$

where $\tau$ is a term. It will be enough to find a condition $p$ extending $(f, \vec{X})$ and a map $h \in M$ with $\operatorname{dom}(h)=\gamma$ so that $p \Vdash \operatorname{ran}(\tau) \subseteq \operatorname{ran}(h)$. Lemma 2.1.5 gives the desired $p$ and $h$ if $\gamma<\omega_{1}$, so assume $\gamma \geqslant \omega_{1}$.

Let $f \in T_{0, \alpha}$. Let $\alpha<\beta<\omega_{1}$ and $\delta<\gamma$. For any $g$ with $\hat{f} \hat{g} \in X_{\beta}$ we define

$$
\begin{aligned}
\eta_{\delta, g} & =\text { the unique } \eta<\kappa \text { such that } \exists \vec{Y}((\hat{f} \hat{g}, \vec{Y}) \Vdash \tau(\check{\delta})=\check{\eta}), \\
& =0 \text { if no such } \eta \text { exists. }
\end{aligned}
$$

(At most one $\eta$ has the property in question.) Now let

$$
S_{\delta, \beta}=\left\{\eta<\kappa \mid \eta=\eta_{\delta, g} \text { for nonmeager many } g \in T_{\alpha, \beta}\right\} \text {. }
$$

Then $S_{\delta, \beta}$ is countable since any family of disjoint nonmeager sets in $T_{\alpha, \beta}$ is countable. Also, for comeager many $g \in T_{\alpha, \beta}$ we have $\eta_{\delta, g} \in S_{\delta, \beta}$ (by 1.1 .3 a wellordered union of meager sets is meager). Thus we can set

$$
Y_{\beta}=\left\{\hat{f} g \in X_{\beta} \mid \forall \delta<\gamma\left(\eta_{\delta, g} \in S_{\delta, \beta}\right)\right\},
$$

and $Y_{\beta}=\varnothing$ for $\beta \leqslant \alpha$. Now $\left\{g \mid \hat{f} g \in Y_{\beta}\right\}$ is a wellordered intersection of sets comeager in $T_{\alpha, \beta}$, so comeager in $T_{\alpha, \beta}$ by 1.1 .3 . Thus $(f, \vec{Y})$ is a condition. Clearly $(f, \vec{Y}) \leqslant(f, \vec{X})$ and $\forall \delta<\gamma$

$$
(f, \vec{Y}) \Vdash \tau(\check{\delta}) \in \bigcup_{\beta<\omega_{1}} S_{\delta, \beta} .
$$

Let $h(\delta, \beta, \zeta)$ be the $\zeta$ th element in the increasing enumeration of $S_{\delta, \beta}$. Then $h \in M$ (it is defined without AC) and $h$ maps a subset of $\gamma \times \omega_{1} \times \omega_{1}$ onto $\bigcup_{\delta<\gamma} \cup_{\beta<\omega_{1}} S_{\delta, \beta}$. Since $\gamma \geqslant \omega_{1}$, we may assume $\operatorname{dom}(h)=\gamma$. Then $p=(f, \vec{Y})$ and $h$ are as desired.

LEMMA 2.1.7. $M^{*} \vDash \exists f \forall \alpha<\omega_{1}(f(\alpha)$ maps $\omega$ onto $\alpha)$.

Proof. Let $g=\cup\{f \mid \exists \vec{X}((f, \vec{X}) \in G)\}$. By density, $\operatorname{dom}(g)=\omega_{1}^{M}$. Since $\omega_{1}^{M}=$ $\omega_{1}^{M^{*}}$, from $g$ we get the desired $f$.

We now add $M \vDash A_{C_{R}}$ to our hypotheses on $M$, and thereby obtain some choice principles in $M^{*}$. The key is that if $M \vDash A C_{\mathrm{R}}$ then the forcing language is full.

LEMMA 2.1.8. Suppose also $M \vDash A C_{\mathbf{R}}$. Then if $p \Vdash \exists v \varphi(v)$, then $p \Vdash \varphi(\tau)$ for some term $\tau$. 
Proof. We work in $M$. Let $p=(f, \vec{X})$. Part 3(a) of the definition of $P$ implies that there is a one-one map of $\bigcup_{\alpha \leqslant \beta<\omega_{1}} X_{\beta+1}$ into $\mathbf{R}$, where $\alpha=\operatorname{dom}(f)$. Applying $\mathrm{AC}_{\mathbf{R}}$, we have functions

$$
g \mapsto \vec{Y}_{g}, \quad g \mapsto \tau_{g},
$$

defined for those $g$ such that $f \subseteq g$ and $\exists \beta\left(g \in X_{\beta+1}\right)$ and $\exists \vec{Y} \exists \tau((g, \vec{Y}) \Vdash \varphi(\tau))$, with the property

$$
\left(g, \vec{Y}_{g}\right) \Vdash \varphi\left(\tau_{g}\right) .
$$

Let $r_{g}=\left(g, \vec{Y}_{g}\right)$.

Now we can define the term $\tau$. Roughly, $\tau$ waits for a condition $r_{g}$ to appear in the generic object (one must by density). If $g$ is of minimal length such that $r_{g}$ appears, then $\tau$ acts like $\tau_{g}$.

Precisely, we put $(q, \sigma)$ into $\tau$ iff for some $g$

$$
r_{g} \text { is defined } \wedge q \leqslant r_{g} \text {. }
$$

$\forall \gamma<\operatorname{dom}(g)\left(r_{g \mid \gamma}\right.$ is defined $\Rightarrow q$ is incompatible with $\left.r_{g \mid \gamma}\right)$, and

$$
\exists s \geqslant q\left((s, \sigma) \in \tau_{g}\right) .
$$

It is easy to check that $p \Vdash \varphi(\tau)$.

LEMMA 2.1.9. If $M \vDash A C_{\mathbf{R}}$, then $M^{*} \vDash A C_{\mathbf{R}}$ and $M^{*} \vDash D C$.

PRoof. Suppose $p \Vdash \forall x \in \mathbf{R} \exists y \varphi(x, y)$. Then by 2.1.8, $\forall x \in \mathbf{R} \exists \tau(\tau$ is a term $\wedge p \Vdash \varphi(\check{x}, \tau))$. Using $\mathrm{AC}_{\mathbf{R}}$ in $M$, we pick for each $x \in \mathbf{R}$ a witness term $\tau_{x}$. For terms $\rho, \tau$, let $[\rho, \tau]$ be a term for the pair of sets named by $\rho$ and $\tau$, and let $\boldsymbol{\sigma}=\left\{\left(q,\left[\check{x}, \tau_{x}\right]\right) \mid q \in P \wedge x \in \mathbf{R}\right\}$. It follows that $p \Vdash \forall x \in \mathbf{R} \varphi(x, \sigma(x))$.

$\mathrm{DC}$ is proved similarly.

We could also have shown $M^{*}$ FDC under the additional assumption that $M \vDash V=L(\mathbf{R})$.

LEMMA 2.1.10. Suppose $M \vDash Z F+A D+A C_{\mathbf{R}}+V=L(\mathbf{R} \cup P(\mathbf{R}))$. Then $M^{*}$ F $\omega_{1}-D C$.

Proof. Suppose $Q, S \in M^{*}$ and

$$
M^{*} \vDash \forall \alpha<\omega_{1} \forall f: \alpha \rightarrow S \exists y \in S((f, y) \in Q) .
$$

We will first find in $M^{*}$ an $S^{\prime} \subseteq S$ so that (i) holds with $S^{\prime}$ replacing $S$, and $S^{\prime}$ is the surjective image of $\mathbf{R}$ in $M^{*}$.

Fix terms $\dot{S}, \dot{Q}$ denoting $S$ and $Q$ respectively. Let $p \in G$ and

$$
p \Vdash \forall \alpha<\omega_{1} \forall f: \alpha \rightarrow \dot{S} \exists y \in \dot{S}((f, y) \in \dot{Q}) .
$$

We work in $M$ for a while now. Since $V=L(\mathbf{R} \cup P(\mathbf{R}))$, there is a definable map $u$ of $O R \times \mathbf{R} \times P(\mathbf{R})$ onto $V$. We define now a strictly increasing sequence $\left\langle\kappa_{\beta}\right| \beta<$ $\left.\omega_{1}\right\rangle$ of ordinals $<\theta$, and a sequence $\left\langle A_{\beta} \mid \beta<\omega_{1}\right\rangle$ of sets of ordinals each of order type $<\theta$. Let $T_{\beta}=\left\{u(\eta, x, B) \mid \eta \in A_{\beta} \wedge x \in \mathbf{R} \wedge O T_{w}(B)<\kappa_{\beta}\right\}$. Choose $\kappa_{0}$ and $A_{0}$ large enough that $\varnothing \in T_{0}$. For $\lambda$ a limit, let $\kappa_{\lambda}=\sup \left\{\kappa_{\beta} \mid \beta<\lambda\right\}$ and $A_{\lambda}=$ $\cup_{\beta<\lambda} A_{\beta}$. Finally, suppose $\kappa_{\beta}$ and $A_{\beta}$ are given. Since $\left\{f \mid \exists \alpha<\omega_{1}\left(f: \alpha \rightarrow T_{\beta}\right)\right\}$ is 
the surjective image of $\mathbf{R}$, and since $\theta$ is regular, there is a $\kappa^{\prime}<\theta$ so that if $\alpha<\omega_{1}$ and $\left\langle\tau_{\delta}\right| \delta\langle\alpha\rangle$ is a sequence of terms in $T_{\beta}$, then there is a term $\tau$ such that

$\varnothing \Vdash \tau$ is a function with domain $\check{\alpha}$,

$$
\varnothing \Vdash \tau(\check{\delta})=\tau_{\delta} \text {, for each } \delta<\alpha,
$$

and

$$
\tau=u(\eta, x, B) \text { for some } B \text { with } O T_{w}(B)<\kappa^{\prime} .
$$

Moreover, we can effectively find $A^{\prime} \supseteq A_{\beta}$ so that $A^{\prime}$ has order type $<\theta$, and for each $\left\langle\tau_{\delta}\right| \delta\langle\alpha\rangle$ as above, there is a term $\tau=u(\eta, x, B)$ as above, with $\eta \in A^{\prime}$. Let $\kappa_{\beta+1}>\kappa^{\prime}$ and $A_{\beta+1} \supseteq A^{\prime}$ be large enough that if $\tau$ is a term, $\tau \in T_{\beta}$, then for some term $\sigma \in T_{\beta+1}$,

$$
p \Vdash \exists \alpha<\omega_{1}(\tau: \alpha \rightarrow \dot{S}) \Rightarrow[(\tau, \sigma) \in \dot{Q} \wedge \sigma \in \dot{S}] .
$$

By 2.1.8, such a $\sigma$ exists. We can effectively find such a $\kappa_{\beta+1}<\theta$ and $A_{\beta+1}$ of order type $<\theta$ because $T_{\beta}$ is the surjective image of $\mathbf{R}$ and $\theta$ is regular. (Recall $\theta=$ $\sup \left\{O T_{w}(B) \mid B \subseteq \mathbf{R}\right\}$; cf. the proof is 1.3.2.)

We move back to $M^{*}$. Let $S^{\prime}$ be the set of denotations of terms $\tau \in \cup_{\beta<\omega_{1}} T_{\beta}$ such that $q \Vdash \tau \in \dot{S}$ for some $q \in G$. The construction and the fact that ${ }^{\omega} M \cap M^{*} \subseteq M$ guarantee that (i) holds with $S^{\prime}$ replacing $S$. Since $\theta$ is regular, $\sup \left\{\kappa_{\beta} \mid \beta<\omega_{1}\right\}<\theta$ and $\cup_{\beta<\omega_{1}} A_{\beta}$ has order type $<\theta$, and thus $\cup_{\beta<\omega_{1}} T_{\beta}$ is the surjective image of $\mathbf{R}$. Thus $S^{\prime}$ is the surjective image of $\mathbf{R}$.

We may then assume that $S^{\prime}=\mathbf{R}$. But then 2.1.7 gives a one-one map $i$ : $\left(\cup_{\beta<\omega_{1}}^{\beta} \mathbf{R}\right) \rightarrow \mathbf{R}$. Let $F: \mathbf{R} \rightarrow \mathbf{R}$ be such that $\forall x \in \mathbf{R}\left(i^{-1}(x)\right.$ exists $\Rightarrow$ $\left.\left(i^{-1}(x), F(x)\right) \in Q\right)$; such an $F$ exists by Unif $(\mathbf{R} \times \mathbf{R})$ in $M^{*}$. Define $f: \omega_{1} \rightarrow \mathbf{R}$ by: $f(\alpha)=F(i(f \uparrow \alpha))$. Clearly, $f$ is the required $\omega_{1}$-DC sequence.

Theorem 2.1.1 has been proved.

At the moment we do not see how to replace the use of Baire category with that of Lebesgue measure in the proof of 2.1.1. If $M \vDash Z F+D C+$ All sets of reals are Lebesgue measurable, can one construct a generic extension $M^{*}$ of $M$ satisfying 2.1.1(a), (b) and (c)?

There are some variants of the model $M^{*}$ of lesser interest. Given $M \vDash Z F+D C$ + All sets have the property of Baire, we can force over $M$ with conditions analogous to those used above, except that the space $T_{\alpha, \beta}$ is replaced by $\times_{\alpha \leqslant \delta<\beta}{ }^{\omega} \omega$. This forcing adds a sequence $\left\langle x_{\alpha} \mid \alpha<\omega_{1}\right\rangle$ of reals; if we let $M^{\prime}=M\left(\left\{x_{\alpha} \mid \alpha<\omega_{1}\right\}\right)$, then $M^{\prime}$ ₹ every wellordered set of reals is countable, but $M^{\prime} \forall\left\{x_{\alpha} \mid \alpha<\omega_{1}\right\}$ has the Baire property. A dual forcing gives a model $M^{\prime \prime}$ where all wellordered sets of reals are countable but there is a non-Lebesgue measurable set. Neither the $M^{\prime}$ forcing nor the $M^{\prime \prime}$ forcing adds reals or collapses cardinals. Finally, it is possible to generalize these notions of forcing so as to add an $\omega_{2}$-sequence of reals without adding new reals or collapsing cardinals. We do not know how to add a $\kappa$-sequence of reals, for $\kappa>\omega_{2}$, without adding reals or collapsing cardinals.

Corollary 2.1.11. Suppose $Z F+A D+A C_{\mathbf{R}}$ is consistent. Then $Z F C+A D^{L(\mathbf{R})}$ $+\delta_{2}^{1}=\omega_{2}$ is consistent. 
Proof. Let $M$ be a countable transitive model of $\mathrm{ZF}+\mathrm{AD}+\mathrm{AC}_{\mathbf{R}}+\mathrm{V}=$ $L(\mathbf{R} \cup P(\mathbf{R}))$ and let $M^{*}$ be the generic extension of $M$ given by 2.1.1. In $M^{*}$ let $P=\left\{h \mid \exists \alpha<\omega_{2}(h: \alpha \rightarrow \mathbf{R})\right\}$, and let $\mathbf{P}=(P, \supseteq)$. Let $G$ be P-generic over $M^{*}$. Clearly $\mathbf{P}$ is $\omega_{1}$-closed, so DC in $M^{*}$ implies that $\mathbf{R}^{M^{*}}=\mathbf{R}^{M^{*}[G]}$ and $\omega_{1}$-DC in $M^{*}$

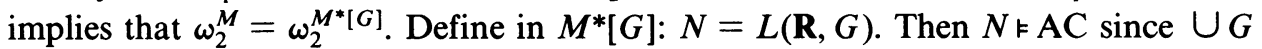
maps $\omega_{2}$ onto $\mathbf{R}^{N}$. Since $\mathbf{R}^{N}=\mathbf{R}^{M}$, we have $N=\mathrm{AD}^{L(\mathbf{R})}$ and $\left(\boldsymbol{\delta}_{2}^{1}\right)^{N}=\left(\boldsymbol{\delta}_{2}^{1}\right)^{M}$. Clearly $\omega_{2}^{M} \geqslant \omega_{2}^{N} \geqslant \omega_{2}^{L(\mathbf{R})}$, but also $\omega_{2}^{M}=\omega_{2}^{L(\mathbf{R})}$ by 1.2.4. Thus $\omega_{2}^{M}=\omega_{2}^{N}$. We have then $N \vDash \mathrm{ZFC}+\mathrm{AD}^{L(\mathbf{R})}+\boldsymbol{\delta}_{2}^{1}=\omega_{2}$.

In $\S 3$ we shall show that the following is true in the model $N$ just constructed.

(*) $\forall f: \omega_{1} \rightarrow \omega_{1} \exists g: \omega_{1} \rightarrow \omega_{1}(\forall \alpha(f(\alpha) \leqslant g(\alpha)) \wedge \exists x \in \mathbf{R}(g \in L[x]))$.

In ZFC + “All $\Pi_{1}^{1}$ games are determined" $+(*)$ one can prove that $\delta_{2}^{1}=\omega_{2}$.

As we saw in 1.2.3, the existence of generic extensions satisfying choice of ground models satisfying $\mathrm{AD}$ gives information about the ground models. For example

Corollary 2.1.12. Assume $Z F+A D+D C$. Then $\forall \kappa\left(\omega_{1} \leqslant \kappa<\theta \Rightarrow \operatorname{cof}\left(\kappa^{+}\right) \geqslant\right.$ $\left.\omega_{2}\right)$.

Proof. We work for a moment in ZF + AD + DC. Suppose $\omega_{1} \leqslant \kappa<\theta$ and $\left\langle\lambda_{\alpha} \mid \alpha<\omega_{1}\right\rangle$ is a strictly increasing sequence with limit $\kappa^{+}\left(\operatorname{cof}\left(\kappa^{+}\right) \geqslant \omega_{1}\right.$ by DC). By playing a Solovay-style game (cf. Theorem 7.1 of [4]) we have a function $G$ so that if $f: \omega \stackrel{\text { onto }}{\rightarrow} \alpha$, then $G(f): \kappa \rightarrow \kappa^{+}$and $\lambda_{\alpha} \subseteq \operatorname{ran}(G(f))$.

Now let $M$ be a countable transitive model of a sufficiently large fragment of $\mathrm{ZF}+\mathrm{AD}+\mathrm{DC}$, and let $M^{*}$ be as in 2.1.1. Suppose that the situation of the last paragraph occurs in $M$. In $M^{*}$ we have $\left\langle f_{\alpha} \mid \alpha<\omega_{1}\right\rangle$ so that $f_{\alpha}: \omega \stackrel{\text { onto }}{\rightarrow} \alpha$. Then if $H(\alpha, \beta)=G\left(f_{\alpha}\right)(\beta)$, then $H$ maps $\omega_{1} \times \kappa$ onto $\left(\kappa^{+}\right)^{M}$. This contradicts 2.1.1(b).

Martin has shown that $\mathrm{ZF}+\mathrm{AD}+\mathrm{DC}$ proves $\forall n \geqslant 2\left(\operatorname{cof}\left(\omega_{n}\right)=\omega_{2}\right)$. We believe the proof of 2.1 .12 will someday generalize to show, in $\mathrm{ZF}+\mathrm{AD}+\mathrm{DC}$, that $\omega_{\omega} \leqslant \kappa<\theta \Rightarrow \operatorname{cof}\left(\kappa^{+}\right) \geqslant \omega_{\omega+1}$.

An argument similar in spirit to that of 2.1.12 answers a question of [1]. Namely, assuming $\mathrm{ZF}+\mathrm{AD}_{\mathbf{R}}+\boldsymbol{\theta}$ is regular, one can show that the two supercompactness measures on $\mathbf{P}_{\omega_{1}}\left(\omega_{2}\right)$ defined in [1] are both identical to the measure defined (implicitly) in $[8, \S 3]$. We omit the proof.

3. The nonstationary ideal on $\omega_{1}$ in $N$. The first link between determinacy and large cardinal ideas was the result of Solovay-Theorem 1.1.11-that $\omega_{1}$ is measurable, granted AD. Later results of the same sort include the measurability of each $\boldsymbol{\delta}_{n}^{1}$ and Moschovakis' result, Theorem 1.1.5. Any of these can be used to show the consistency of ZFC + 'there exists a measurable cardinal' by a standard relative constructibility argument, but more can be accomplished by other means. Work of John Green has established, from the consistency of $\mathrm{ZF}+\mathrm{DC}+\boldsymbol{\Delta}_{2}^{1}$-determinacy, the consistency of $\mathrm{ZFC}+$ 'there is a cardinal with a normal measure concentrating on measurables'.

For the record, an ideal $I$ of subsets of $\kappa$ is $\lambda$-saturated iff $P(\kappa) / I$ has the $\lambda$-chain condition. The theory of saturation of ideals has a long record of intimacy with that 
of large cardinals, for the details of which one should consult Kunen [5]. The best relative consistency results between the theories of saturated ideals on $\omega_{1}$ and large cardinals are the theorem of Kunen [5] that the consistency of ZFC + "there is a huge cardinal" implies that of ZFC + "there is an $\omega_{2}$-saturated ideal on $\omega_{1}$ ", and the result of Mitchell [7] that the consistency of ZFC + "there is an $\omega_{2}$-saturated ideal on $\omega_{1}$ " implies that of ZFC + "there is a cardinal $\kappa$ which is $P^{2}(\kappa)$-measurable".

In this section we shall show that the ideal $N S_{\omega_{1}}$ of nonstationary subsets of $\omega_{1}$ is $\omega_{2}$-saturated in the model $N$ of 2.1.11. This settles a question left open by the Kunen result quoted above, and provides consistency strength consequences of determinacy hypotheses in the theory of saturated ideals.

H. Woodin has recently constructed, from a ground model of $\mathrm{ZF}+\mathrm{AD}_{\mathbf{R}}+\boldsymbol{\theta}$ is regular, a model of ZFC with an ideal $I$ on $\omega_{1}$ so that $P\left(\omega_{1}\right) / I$ has a dense set of size $\omega_{1}$. This property of $I$ is ostensibly stronger than $\omega_{2}$-saturatedness. Woodin's ideal is not $N S_{\omega_{1}}$ (cf. [10]).

Finally, our proof easily implies that Chang's conjecture does not follow from the assumption that $N S_{\omega_{1}}$ is $\omega_{2}$-saturated.

Throughout this section, $M$ and $M^{*}$ are the models of 2.1.1. We assume $M$ satisfies all the hypotheses of 2.1.1. The first step is to identify $N S_{\omega_{1}}$ in $M^{*}$ with the ideal generated by the nonstationary ideal on $\omega_{1}$ in the sense of $M$. This is accomplished by the following lemma.

Lemma 3.1.1. Suppose $\psi: \omega_{1} \rightarrow \omega_{1}$ and $\psi \in M^{*}$. Then $\exists \varphi: \omega_{1} \rightarrow \omega_{1}(\varphi \in M \wedge \forall \alpha$ $(\psi(\alpha) \leqslant \varphi(\alpha)))$. Thus every cub subset of $\omega_{1}$ in $M^{*}$ has a cub subset in $M$.

Proof. To prove the first assertion, let $p=\langle f, \vec{X}\rangle$ be a condition with $f \in T_{0, \alpha}$, and let $\tau$ be a term such that $p \Vdash \tau: \check{\omega}_{1} \rightarrow \check{\omega}_{1}$. It suffices to find $q \leqslant p$ and $\varphi: \omega_{1} \rightarrow \omega_{1}$ in $M$ so that $\forall \alpha<\omega_{1}(q \Vdash \tau(\check{\alpha})<\varphi(\check{\alpha}))$.

Working in $M$, let $\beta<\omega_{1}$ be given. By 2.1.4 we can find $\alpha<\gamma_{\beta}<\omega_{1}$ so that for almost all $g \in T_{\alpha, \gamma_{\beta}}, \exists \vec{Y} \exists \delta(\langle\dot{f}, \vec{Y}\rangle \Vdash \tau(\check{\beta})=\check{\delta})$. By 1.1 .3 we can fix $\delta_{\beta}<\omega_{1}$ so that $S_{\beta}=\left\{g \in T_{\alpha, \gamma_{\beta}} \mid \exists \vec{Y}\left(\left\langle f^{\hat{g}} g, \vec{Y}\right\rangle \Vdash \tau(\check{\beta})<\check{\delta_{\beta}}\right)\right\}$ is comeager. Now for $\gamma>\alpha$ let

$$
\begin{aligned}
Z_{\gamma}=\left\{g \in X_{\gamma} \mid \forall \beta<\omega_{1}\left(\gamma_{\beta} \leqslant\right.\right. & \gamma \Rightarrow g \uparrow\left(\gamma_{\beta}-\alpha\right) \in S_{\beta} \wedge \\
& \left.g \uparrow\left(\gamma-\gamma_{\beta}\right) \in F\left(g \uparrow\left(\gamma_{\beta}-\alpha\right), \vec{X}, “ \tau(\check{\beta})<\check{\delta_{\beta}} ”\right)\right\} .
\end{aligned}
$$

(Here $F$ is the function of 2.1 .2 which picks a restriction forcing a given statement.) Using 1.1.2 and 1.1.3 we can see that $\left\{g \in T_{\alpha, \delta} \mid f^{\hat{\prime}} g \in Z_{\delta}\right\}$ is comeager. Let $Z_{\delta}=X_{\delta}$ for $\delta \leqslant \alpha$. Then $q=\langle f, Z\rangle$ is a condition, $q \leqslant p$, and if $\varphi(\beta)=\delta_{\beta}$ for $\beta<\omega_{1}$, we have $q$ ॥ $\tau(\breve{\beta})<\varphi(\breve{\beta})$ for $\beta<\omega_{1}$.

Now if $\varphi$ pointwise dominates the increasing enumeration of some cub $C \subseteq \omega_{1}$, then $\left\{\alpha \mid \varphi^{\prime \prime} \alpha \subseteq \alpha\right\}$ is a cub subset of $C$. The second assertion of 3.1 .1 follows at once.

By a similar argument we can also show that if $U$ is a $\kappa$-complete ultrafilter on an ordinal $\lambda$ in $M$, where $\kappa \geqslant \omega_{2}$, then $U$ generates a $\kappa$-complete ultrafilter on $\lambda$ in $M^{*}$. For let $p \Vdash \dot{A} \subseteq \lambda$ for some term $\dot{A}$, where $p=\langle f, \vec{X}\rangle$ and $f \in T_{0, \alpha}$. For each $\beta<\lambda$ there is $\eta_{\beta}>\alpha$ so that $S_{\beta}=\left\{g \in T_{\alpha, \eta_{\beta}} \mid \exists \vec{Y}\left(\left\langle f^{\hat{\prime}} g, \vec{Y}\right\rangle\right.\right.$ decides $\left.\left.\beta \in \dot{A}\right)\right\}$ is comeager 
(by 2.1.4). Fix $\gamma$ so that for $U$ a.e. $\beta<\lambda, \gamma=\eta_{\beta}$. By 1.1.3 fix $g \in T_{\alpha, \gamma}$ so that $\gamma=\eta_{\beta} \Rightarrow g \in S_{\beta}$. By symmetry, we may assume that for $U$ a.e. $\beta\langle\lambda, \exists \hat{Y}(\langle\hat{f} \hat{g}, \vec{Y}\rangle$ $\Vdash \check{\beta} \in \dot{A})$. For each such $\beta$ pick a $\vec{Y}^{\beta}$ witnessing this using 2.1.2. Let $Z_{\delta}=\bigcap_{\beta} Y_{\delta}^{\beta} \cap$ $X_{\delta}$. Then $\langle f, \vec{Z}\rangle \leqslant p$, and there is a set $B \in U$ so that $\langle f, \vec{Z}\rangle \Vdash \check{\beta} \subseteq A$. The $\kappa$-completeness of the ultrafilter generated by $U$ in $M^{*}$ can be shown similarly.

We refer hereafter to the nonstationary ideal on $\omega_{1}$ in $M^{*}$ as $I$. By $I^{+}$we mean the set of stationary subsets of $\omega_{1}$ in $M^{*}$. We say that a subset of $\omega_{1}$ in $M$ is large if it includes a cub set, and small if it is disjoint from a cub set. By 1.1.11, every subset of $\omega_{1}$ is either large or small.

LEMMA 3.1.2. In $M^{*}$ there is an isomorphism between $P\left(\omega_{1}\right) / I$ and $R O(\mathbf{Q})$.

Proof. Working in $M^{*}$, we define a map $B: \mathbf{Q} \rightarrow P\left(\omega_{1}\right) / I-\{0\}$. Let $q \in \mathbf{Q}$ be given. Thus $q$ : $\left(\omega_{2}-\omega_{1}\right) \times \omega \rightarrow \omega_{2}$ and $q$ is finite. Since $q$ is finite, $q \in M$. By 1.1.11 we can find in $M$ a sequence $\left\langle q_{\alpha} \mid \alpha<\omega_{1}\right\rangle$ which represents $q$ in the ultrapower of $M$ by the closed unbounded filter on $\omega_{1}$ in $M$. Now kos' theorem holds for this ultrapower for formulae whose quantifiers range over wellordered sets, and so we have a cub $C \in M$ so that for $\alpha \in C$

$$
q_{\alpha}:\left(\omega_{1}-\alpha\right) \times \omega \rightarrow \omega_{1}
$$

and

$$
q_{\alpha}(\beta, n)<\beta
$$

and

$$
q_{\alpha} \text { is finite. }
$$

Now let $F=\cup\{f \mid \exists \vec{X}((f, \vec{X}) \in G)\}$ where $M^{*}=M[G]$. Thus $F: \omega_{1} \times \omega \rightarrow \omega_{1}$. By a density argument, $\left\{\alpha \mid q_{\alpha} \subseteq F\right\}$ meets every unbounded subset of $C$ in $M$, and so by 3.1 .1 is stationary in $M^{*}$. We set

$$
B(q)=\left\{\alpha \mid q_{\alpha} \subseteq F\right\} / I .
$$

To see that $B(q)$ is well defined, notice that if $\left\langle r_{\alpha} \mid \alpha<\omega_{1}\right\rangle$ also represents $q$, then $r_{\alpha}=q_{\alpha}$ for all $\alpha$ in some cub $C$, and so $\left\{\alpha \mid r_{\alpha} \subseteq F\right\} / I=\left\{\alpha \mid q_{\alpha} \subseteq F\right\} / I$.

To see that $B$ is order preserving, let $r \leqslant q$ in $\mathbf{Q}$. Then $q_{\alpha} \subseteq r_{\alpha}$ for all $\alpha$ in some cub $C$ (by the restricted kos theorem). So $\left\{\alpha \mid r_{\alpha} \subseteq F\right\} \cap C \subseteq\left\{\alpha \mid q_{\alpha} \subseteq F\right\}$ and $B(r) \leqslant B(q)$. On the other hand, if $r \neq q$ then $q_{\alpha} \nsubseteq r_{\alpha}$ for all $\alpha$ in some cub $C \in M$. By density, $\left\{\alpha \mid r_{\alpha} \subseteq F \wedge q_{\alpha} \nsubseteq F\right\}$ meets every unbounded subset of $C$ in $M$. By 3.1.1, $B(r) \$ B(q)$.

We next show that $\operatorname{ran}(B)$ is dense in $P\left(\omega_{1}\right) / I-\{0\}$. Let $D / I$ be nonzero. Let $\dot{D}$ be a name for $D$ and pick any $p \in G$ so that $p \Vdash \dot{D}$ is stationary. Let $p=\langle f, \vec{X}\rangle$, where $f \in T_{0, \delta}$. Working now in $M$ we shall find $r \leqslant p$ in $P$ and $q \in \mathbf{Q}$ so that $r \Vdash B(q) \leqslant D / I$. By density, some such $r$ is in $G$, and we are done.

In $M$ we define, for $\delta<\alpha<\gamma<\omega_{1}$,

$$
\begin{aligned}
& S_{\alpha, \gamma}=\left\{g \in T_{\delta, \alpha} \mid\left\{h \in T_{\alpha, \gamma} \mid \exists \vec{Y}((\hat{f \hat{g} h}, \vec{Y}) \leqslant(f, \vec{X}) \wedge\right.\right. \\
& (\hat{f} \hat{g} h, \vec{Y}) \Vdash \check{\alpha} \in \dot{D})\} \text { is comeager }\} \text {. }
\end{aligned}
$$

Notice that $S_{\alpha, \gamma} \subseteq S_{\alpha, \gamma^{\prime}}$ if $\gamma<\gamma^{\prime}$. 
Claim. $\left\{\alpha \mid \exists \gamma\left(S_{\alpha, \gamma}\right.\right.$ is comeager) $\}$ contains a cub.

Proof. Otherwise, let $C$ be cub so that $\alpha \in C \Rightarrow \forall \gamma\left(S_{\alpha, \gamma}\right.$ is not comeager). For $\alpha \in C$ and $\gamma>\alpha$, let $\left[t_{\alpha, \gamma}\right]$ be an interval on which $S_{\alpha, \gamma}$ is meager. Let $t_{\alpha}=t_{\alpha, \gamma}$ for cofinally many $\gamma$. Then $S_{\alpha, \gamma}$ is meager on $t_{\alpha}$ for all $\gamma$, so $\cup_{\gamma<\omega_{1}} S_{\alpha, \gamma}$ is meager on $t_{\alpha}$. Now, using Födor's theorem, we may assume that for some fixed $t, \alpha \in C \Rightarrow t_{\alpha}=t$. Using 1.1.2 and 1.1.3 it is easy to define a condition $(f, \vec{Z}) \leqslant(f, \vec{X})$ so that for $\alpha \in C, \hat{f} g \in Z_{\alpha}$, and $t \subseteq g$ we have

$$
g \notin \underset{\gamma<\omega_{1}}{ } S_{\alpha, \gamma},
$$

and

$$
\forall \gamma>\alpha \forall h\left(\hat{f \hat{g} h} \in Z_{\gamma} \Rightarrow \nexists \vec{Y}((\hat{f} \hat{g} h, \vec{Y}) \Vdash \check{\alpha} \in \dot{D})\right) \text {. }
$$

Let $t \subseteq g$ and $(\hat{f} \hat{g}, \vec{Z}) \leqslant(f, \vec{Z})$. Then $(\hat{f} g, \vec{Z}) \Vdash \dot{D} \cap \check{C}=\varnothing$, a contradiction proving the claim.

So let $S_{\alpha, \gamma_{\alpha}}$ be comeager for all $\alpha \in C$, where $C$ is a cub. For $\alpha \in C$, let $\left[q_{\alpha}\right]$ be an interval of $T_{\alpha, \gamma_{\alpha}}$ so that for comeager many $g \in T_{\gamma, \alpha},\left\{h \in T_{\alpha, \gamma_{\alpha}} \mid \exists \vec{Y}\left(\left(f^{\hat{g}} \hat{g} h, \vec{Y}\right) \Vdash \alpha\right.\right.$ $\in \dot{D})\}$ is comeager on $\left[q_{\alpha}\right]$. Let $q$ be represented by $\left\langle q_{\alpha}\right| \alpha\left\langle\omega_{1}\right\rangle$ in the ultrapower of $M$ by the cub filter. Thus $q \in \mathbf{Q}$, as desired.

By our standard manipulations (using 1.1.2 and 1.1.3) we can find $(f, \vec{Z}) \leqslant(f, \vec{X})$ so that whenever $\alpha \in C, \hat{f^{\hat{g}} h} \in Z_{\gamma_{\alpha}}$ and $q_{\alpha} \subseteq h$ we have

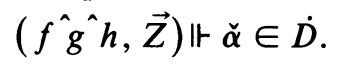

Set $r=(f, \vec{Z})$. Then for $\alpha \in C$

$$
r \Vdash q_{\alpha} \subseteq \dot{F} \Rightarrow \alpha \in \dot{D},
$$

so

$$
r \Vdash B(q) \leqslant \dot{D} / I,
$$

as desired.

Thus $P\left(\omega_{1}\right) / I-\{0\}$ has a dense subset isomorphic to $\mathbf{Q}$. To finish the proof, we must show that $P\left(\omega_{1}\right) / I$ is complete in $M^{*}$. Since we do not have the full axiom of choice in $M^{*}$, we sketch this otherwise standard proof.

It suffices to show that suprema exist for subsets of the canonical dense set which we shall identify with $\mathbf{Q}$. Since $\mathbf{Q}$ is wellorderable and has the $\omega_{2}$-chain condition, it suffices to find the supremum of a family $\left\langle b_{\alpha} \mid \alpha<\omega_{1}\right\rangle$ of disjoint members of $\mathbf{Q}$. Let $\left\langle a_{\alpha} \mid \alpha<\omega_{1}\right\rangle$ extend $\left\langle b_{\alpha} \mid \alpha<\omega_{1}\right\rangle$ to a maximal disjoint family in $\mathbf{Q}$, and let $\left\{c_{\alpha} \mid \alpha<\omega_{1}\right\}=\left\{b_{\alpha} \mid \alpha<\omega_{1}\right\} \cup\left\{a_{\alpha} \mid \alpha \subseteq \omega_{1}\right\}$. Pick representatives $C_{\alpha}$ for $c_{\alpha}$. For this we use $\omega_{1}$-AC. Now define $C_{\alpha}^{\prime}=C_{\alpha}-\cup_{\beta<\alpha} C_{\beta}$. Then let $D$ be the union of those $C_{\alpha}^{\prime}$ that came from $b_{\beta}$ 's (as opposed to $a_{\beta}$ 's). $D / I$ is the supremum of $\left\{b_{\alpha} \mid \alpha<\omega_{1}\right\}$.

Now let $N$ be as in Corollary 2.1.11. Since $N$ has the same subsets of $\omega_{1}$ as $M^{*}$ and satisfies $\mathrm{AC}$, we have the following

THEOREM 3.1.3. If $Z F+A D+D C+A C_{\mathbf{R}}$ is consistent, then so is $Z F C+A D^{L(\mathbf{R})}$ + 'the nonstationary ideal on $\omega_{1}$ is $\omega_{2}$-saturated'. 
To handle Chang's Conjecture first notice that no consistent theory of the form ZFC $+\left(\exists A \subseteq \omega_{2}\right) L_{\omega_{2}}[A] \vDash \varphi$ can possibly imply it. For suppose such a theory did imply Chang's Conjecture. Working in the theory let $A$ and $\alpha$ be such that $A \subseteq \alpha$, $\alpha=\omega_{2}^{L[A]}, L_{\alpha}\left[A^{*}\right] \vDash \varphi$, where $A^{*}=\{\beta \mid \beta+1 \in A\}$, and $\omega_{1}^{L[A]}$ has the smallest value it can have for such an $A$ and $\alpha$. Then $L[A]$ satisfies Chang's Conjecture, so $L_{\alpha}[A]$ has an elementary substructure of length greater than or equal to $\omega_{1}^{L[A]}$ but with bounded intersection with $\omega_{1}^{L[A]}$. This collapses to some $L_{\alpha^{\prime}}\left[A^{\prime}\right] \vDash \varphi$, with $A^{\prime} \subseteq \alpha^{\prime}$. Now $\alpha^{\prime} \geqslant \omega_{1}^{L[A]}>\omega_{1}^{L_{\alpha^{\prime}}\left[A^{\prime}\right]}$. By a condensation argument of Hajnal, $L_{\alpha}[A]$ and $L_{\alpha^{\prime}}\left[A^{\prime}\right]$ satisfy the statement "there is only one uncountable cardinal". Thus $\alpha^{\prime}=$ $\omega_{1}^{L[A]}$, and $\alpha^{\prime}$ is regular in $L\left[A^{\prime}\right]$. By Hajnal's theorem again, $\omega_{1}^{L_{\alpha^{\prime}}\left[A^{\prime}\right]}=\omega_{1}^{L\left[A^{\prime}\right]}$. But then $\alpha^{\prime}=\omega_{2}^{L\left[A^{\prime}\right]}$. Hence, $A^{\prime}$ and $\alpha^{\prime}$ satisfy the conditions imposed on $A$ and $\alpha$, but $\omega_{1}^{L\left[A^{\prime}\right]}<\omega_{1}^{L[A]}$.

The model $N$ above could be augmented by the isomorphism of $P \omega_{1} / I$ and $R O(\mathbf{Q})$ which lies in $M^{*}$. Thus we have

Corollary 3.1.4. If $Z F+D C+A D+A C_{\mathbf{R}}$ is consistent, then so is $Z F C+$ $P \omega_{1} / N S_{\omega_{1}} \cong R O(\mathbf{Q})$.

By the preceding argument this latter theory cannot imply Chang's Conjecture if it is consistent. But it does imply the $\omega_{2}$-saturatedness of $N S_{\omega_{1}}$. Hence

CoRollary 3.1.5. If $Z F+D C+A D+A C_{\mathbf{R}}$ is consistent, then $Z F C+N S_{\omega_{1}}$ is $\omega_{2}$-saturated does not imply Chang's Conjecture.

4. Open problems. The first question is whether the hypothesis in our main results (2.1.11 and 3.1.3) is best possible.

(Q.1) Does Con $(\mathrm{ZF}+\mathrm{AD}+\mathrm{DC})$ imply either of $\operatorname{Con}\left(\mathrm{ZFC}+\mathrm{AD}^{L(\mathbf{R})}+\boldsymbol{\delta}_{2}^{1}=\omega_{2}\right)$ or Con(ZFC $+\mathrm{AD}^{L(\mathbf{R})}+N S_{\omega_{1}}$ is $\omega_{2}$-saturated)? ${ }^{1}$

We would regard a positive answer to $Q .1$ as a significant improvement on our main results, because there is a natural candidate for an inner model of $\mathrm{ZF}+\mathrm{AD}+$ DC, namely $L(\mathbf{R})$. No such candidate inner model for $Z F+A D+A_{\mathbf{R}}$ is known.

The presence of $\mathrm{AD}^{L(\mathbf{R})}$ in the conclusion of $\mathrm{Q} .1$ may seem unnatural. In fact, Con(ZFC $+N S_{\omega_{1}}$ is $\omega_{2}$-saturated) is known under no hypothesis other than that we have used. And although L. Harrington has shown that $\operatorname{Con}(\mathrm{ZFC})$ implies Con(ZFC $+\delta_{2}^{1}=\omega_{2}$ ) (cf. [2]), Harrington's method breaks down if one wants even $\Pi_{1}^{1}$-determinacy in the conclusion. (It is natural to consider $\boldsymbol{\Pi}_{1}^{1}$-determinacy in connection with the size of $\delta_{2}^{1}$, since $\Pi_{1}^{1}$-determinacy implies that $\delta_{2}^{1}$ is the second uniform indiscernible [4].) In fact, Martin has shown in unpublished work that Con $\left(\mathrm{ZFC}+\boldsymbol{\Pi}_{1}^{1}\right.$-determinacy $\left.+\boldsymbol{\delta}_{2}^{1}=\omega_{2}\right)$ implies Con $(\mathrm{ZFC}+$ there is a measurable cardinal), so that " $\delta_{2}^{1}=\omega_{2}$ " has strength of its own in this context. We are led to

(Q.2) Can Con(ZFC $+\Pi_{1}^{1}$-determinacy $\left.+\boldsymbol{\delta}_{2}^{1}=\omega_{2}\right)$ be established using any large cardinal or determinacy hypothesis weaker than $\operatorname{Con}\left(\mathrm{ZF}+\mathrm{AD}+\mathrm{AC}_{\mathbf{R}}\right)$ ?

(Q.3) Same as Q.2 for Con(ZFC $+N S_{\omega_{1}}$ is $\omega_{2}$-saturated).

We turn from weakening our hypothesis to strengthening our conclusions. Our forcing method adds to a ground model $M$ of a strong determinacy hypothesis a

${ }^{1} \mathrm{H}$. Woodin (private communication) has answered Question 1 in the affirmative on both counts. 
wellorder of $\mathbf{R}^{M}$ while adding no new reals and preserving $\omega_{2}$. Can we extend the method so as to preserve higher cardinals and cofinalities as well? The next two questions would probably yield to such an extension.

(Q.4) $\operatorname{Con}\left(\mathrm{ZFC}+\mathrm{AD}^{L(\mathbf{R})}+\forall n\left(\boldsymbol{\delta}_{n}^{1}=\omega_{n}\right)\right)$ ?

(Q.5) Con(ZFC + the nonstationary ideal on $\omega_{2}$ is $\omega_{3}$-saturated)?

Such an extension of our forcing seems to require some new descriptive set theory. We have used heavily the existence in $M$ of a map ||$: \mathbf{R} \stackrel{\text { onto }}{\rightarrow} \omega_{1}$ and a sequence $\left\langle I_{\alpha} \mid \alpha<\omega_{1}\right\rangle$ such that $I_{\alpha}$ is an $\omega_{1}$-saturated, wellordered-additive ideal on $\{x \in \mathbf{R}|| x \mid=\alpha\}$. Now fix any map \|\|$: \mathbf{R} \stackrel{\text { onto }}{\rightarrow} \omega_{2}$ in $M$. No sequence $\left\langle J_{\alpha} \mid \alpha<\omega_{2}\right\rangle$ with analogous properties is known. One symptom of the difficulties is that for any extension $M^{* *}$ of $M$ so that $M^{* *} \vDash \exists f: \omega_{2} \rightarrow \mathbf{R} \forall \alpha<\omega_{2}(\|f(\alpha)\|=\alpha)$, we have $M^{* *} \vDash \operatorname{card}\left(\omega_{n}^{M}\right) \leqslant \omega_{2}$ for $n \geqslant 2$. The reason is that $M \vDash \forall n \geqslant 2\left(\operatorname{cof}\left(\omega_{n}\right)=\omega_{2}\right)$. Indeed, by abstracting the essentials from the proof of 2.1 .12 we get

Proposition 4.1. Assume $Z F+A D+D C$. Then there is no $\left\langle J_{\alpha} \mid \alpha<\omega_{2}\right\rangle$ such that $\forall \alpha<\omega_{2}$

(i) $\left(J_{\alpha}\right.$ is an ideal on $\{x \in \mathbf{R} \mid\|x\|=\alpha\}$,

(ii) $\left(J_{\alpha}\right.$ is $\omega_{3}$-additive $)$ and

(iii) ( $J_{\alpha}$ is $\omega_{\omega}$-saturated).

Proof (Sketch). Suppose $\left\langle J_{\alpha} \mid \alpha<\omega_{2}\right\rangle$ were such a sequence. Each $J_{\alpha}$ is $\omega_{\omega+1^{-}}$ additive. Using $D C$ we see that each $J_{\alpha}$ is $\omega_{n}$-saturated for some $n$. Fix $n$ so that for cofinally many $\alpha<\omega_{2}, J_{\alpha}$ is $\omega_{n}$-saturated. We may assume $J_{\alpha}$ is $\omega_{n}$-saturated $\forall \alpha<\omega_{2}$. Fix $g: \omega_{2} \rightarrow \omega_{n+1}$ cofinal. By the coding lemma of [6] and the uniformization theorem for projective relations, there is a function $F:{ }^{\omega} \omega \rightarrow{ }^{\omega} \omega$ so that $\forall x \forall \alpha<\omega_{2}\left(\|x\|=\alpha \Rightarrow F(x)\right.$ codes a map of $\omega_{n}$ onto $\left.g(\alpha)\right)$. Using $\omega_{n}$-saturatedness and $\omega_{\omega}$-additivity in a possible values argument like that of 2.1.6 (but simpler), we get a map of $\omega_{n}$ onto $\omega_{n+1}$.

However, 4.1 is not a serious blow to the program of extending our method to larger cardinals. The natural attempt to define category-type measures on $\{x \mid\|x\|$ $=\alpha\}$ for $\alpha<\omega_{2}$ would involve topologies whose basic intervals are determined by nodes of $T_{2}$, which is a tree on $\omega \times \omega_{\omega}$. (It is the tree constructed implicitly in Theorem 6.3 of [4].) Thus one would only expect $\omega_{\omega+1}$-saturation.

Another limitation on carrying our results further is an unexpected connection between saturation properties of the nonstationary ideals on the $\delta_{n}^{1}$ 's and the conjecture $\forall n\left(\delta_{n}^{1}=\omega_{n}\right)$. Notice here that the nonstationary ideal on $\kappa$ is $\kappa^{+}$-saturated iff $\forall \lambda<\kappa\left(\lambda\right.$ regular $\Rightarrow$ the non- $\lambda$-stationary ideal on $\kappa$ is $\kappa^{+}$-saturated). (Thus Q.5 breaks into two questions.)

Proposition 4.2. Assume $Z F C+A D^{L(\mathbf{R})}+\delta_{3}^{1}=\omega_{3}+\delta_{4}^{1}=\omega_{4}$. Let I be the ideal of non- $\omega_{1}$-stationary subsets of $\omega_{3}$. Then $I$ is not $\omega_{4}$-saturated.

Proof. By $\S 17$ of [4], there are functions $f_{\alpha}: \omega_{3} \rightarrow \omega_{3}$ for $\alpha \leqslant \omega_{1-1}$ so that $\left\{\beta \mid f_{\alpha}(\beta)\right.$ $\left.<f_{\gamma}(\beta)\right\}$ contains an $\omega_{1}$-cub set for $\alpha<\gamma \leqslant \omega_{4}$. Let $g_{\beta}: f_{\omega_{4}}(\beta) \stackrel{1-1}{\rightarrow} \beta$ for $\beta \geqslant \omega_{2}$. Let $A_{\gamma, \delta}=\left\{\beta \mid g_{\beta}\left(f_{\delta}(\beta)\right)=\gamma\right\}$ for $\gamma<\omega_{3}$ and $\delta<\omega_{4}$. 
Notice that $\delta \neq \delta^{\prime} \Rightarrow\left(A_{\gamma, \delta} \cap A_{\gamma, \delta^{\prime}}\right) \in I$. Thus if $I$ is $\omega_{4}$-saturated we have $\delta_{\gamma}<\omega_{4}$ so that $A_{\gamma, \delta} \notin I \Rightarrow \delta<\delta_{\gamma}$. Let $\delta=\sup \left\{\delta_{\gamma} \mid \gamma<\omega_{3}\right\}$. Since $\delta<\omega_{4}, \quad\left\{\beta \mid f_{\delta}(\beta) \in\right.$ $\left.\operatorname{dom}\left(g_{\beta}\right)\right\} \notin I$. Since $I$ is normal, for some fixed $\gamma<\omega_{3}$ we have $\left\{\beta \mid g_{\beta}\left(f_{\delta}(\beta)\right)=\gamma\right\}$ $\notin I$. That is, $A_{\gamma, \delta} \notin I$. But $\delta_{\gamma} \leqslant \delta$, a contradiction.

Proposition 4.2 is due to Martin and, to a lesser extent, to Steel.

Finally, two questions about $N S_{\omega_{1}}$ we do not know how to attack.

(Q.6) Con $\left(\mathrm{ZFC}+P\left(\omega_{1}\right) / N S_{\omega_{1}}\right.$ has a dense subset of size $\left.\omega_{1}\right)$ ?

(Q.7) $\operatorname{Con}\left(\mathrm{ZFC}+N S_{\omega_{1}}\right.$ is $\omega_{2}$-saturated $\left.+\mathrm{CH}\right)$ ?

\section{REFERENCES}

1. C. A. DiPrisco and J. Henle, On the compactness of $\aleph_{1}$ and $\aleph_{2}$, J. Symbolic Logic 43 (1978), 394-402.

2. L. Harrington, Ph.D. thesis, M.I.T., 1973.

3. __ Analytic determinacy and $0^{\#}$, J. Symbolic Logic 43 (1978), 685-694.

4. A. S. Kechris, $A D$ and projective ordinals, Cabal Seminar 76-77, Lecture Notes in Math., vol. 689, Springer-Verlag, Berlin and New York, 1970, pp. 91-133.

5. K. Kunen, Saturated ideals, J. Symbolic Logic 43 (1978), 65-77.

6. Y. N. Moschovakis, Determinacy and prewellorderings of the continuum, Mathematical Logic and Foundations of Set Theory, (Y. Bar-Hillel, editor), North-Holland, Amsterdam and London, 1970, pp. 24-62.

7. W. Mitchell, Hypermeasurable cardinals (to appear).

8. R. Solovay, The independence of $D C$ from $A D$, Cabal Seminar 76-77, Lecture Notes in Math., vol. 689, Springer-Verlag, Berlin and New York, 1970, pp. 171-185.

9. R. Van Wesep, Wadge degrees and descriptive set theory, Cabal Seminar 76-77, Lecture Notes in Math., vol. 689, Springer-Verlag, Berlin and New York, 1970, pp. 151-171.

10. H. Woodin, Ph.D. thesis, Univ. of California, Berkeley, 1980.

Department of Mathematics, University of California, Los ANgeles, California 90024

Department of Mathematics, Goucher College, Towson, Maryland 21204 